\title{
Metal free synthesis of functionalized 1-aryl isoquinolines via iodine mediated oxidative dehydrogenation and ring opening of lactam in isoindoloisoquinolinones
}

\author{
KAMSALI MURALI MOHAN ACHARI, MUTHUPANDI KARTHICK \\ and CHINNASAMY RAMARAJ RAMANATHAN* \\ Department of Chemistry, Pondicherry University, Puducherry 605 014, India \\ E-mail: crrnath.che@ pondiuni.edu.in
}

MS received 6 February 2017; revised 3 April 2017; accepted 5 May 2017

\begin{abstract}
A facile and convenient method for the synthesis of substituted 2-(isoquinolin-1-yl)benzoic acids from isoindoloisoquinolinones in the presence of molecular iodine under sealed tube condition at $100^{\circ} \mathrm{C}$ has been developed. This methodology involves the oxidative dehydrogenation and ring opening of hydroxy lactam/methoxy lactam to furnish the 2-(isoquinolin-1-yl)benzoic acids. Some of these acids are successfully cyclized to furnish the azabenzanthrone derivatives, the potential precursors for the synthesis of menisporphine alkaloids and daurioxoisoaporphines.
\end{abstract}

Keywords. Iodine; oxidative dehydrogenation; oxoisoaporphines; 1-Azabenzanthrone; isoindoloisoquinolinones.

\section{Introduction}

The oxoisoaporphines constitute a small family of alkaloids that are known to occur in the rhizome of Menispermum dauricum DC. (Menispermaceae). The oxoisoaporphine alkaloids possess 1-azabenzanthrone (7 $\mathrm{H}$-dibenzo[de,h]quinolin-7-one) skeleton containing isoquinoline ring incorporated as part of a biaryl system in their structures (A in Figure 1). Derivatives of oxoisoaporphine alkaloids such as daurioxoisoaporphines exhibited cytotoxic activities against a small panel of cancer cell lines. ${ }^{1}$ A series of 9 -amino alkanamido-1-azabenzanthrone derivatives and their quaternary methiodide salts exhibited inhibitory activity against acetylcholine esterase (AChE) and butyrylcholine esterase (BuChE). ${ }^{2}$ The 1-azabenzanthrone moiety of oxoisoaporphine alkaloids displayed DNA binding affinity with calf thymus DNA, ${ }^{3}$ as well as cytotoxicity against tumor cell lines, ${ }^{4}$ antiproliferative activity ${ }^{5}$ and antiplasmodial activity. ${ }^{6}$ Organoplatinum(II) complexes of oxoisoaporphine exhibited antitumor activity. ${ }^{7}$

Synthetic protocols have already been developed to accomplish the synthesis of 1-azabenzanthrone and their derivatives, which are subsequently used to synthesize oxoisoaporphine alkaloids. ${ }^{6-10}$ Oxoisoaporphine alkaloids possess 1-azabenzanthrone skeleton which can be synthesized from the intermediates such as $o$-isoquinolin-2-yl benzoic acid (substituted or unsubstituted) or 2-(3,4-dihydroisoquinolin-1-yl) benzoic acid (substituted or unsubstituted) (Figure 2). Though the intermediates $\mathbf{X}$ and $\mathbf{Y}$ are useful for the synthesis of oxoisoaporphines or dihydrooxoisoaporphines, the methods adopted to prepare these intermediates suffered from one or more disadvantages such as high temperature, multiple steps, tedious workup, long reaction times, anhydrous conditions, low yields and difficult product purification procedures. In view of these drawbacks and our continued effort to show the synthetic potential of TfOH-mediated 6-exo-trig cyclization of phenethylphthalimides, it has become imperative to develop a simple and efficient methodology to convert the isoindoloisoquinolinones to intermediates $\mathbf{X}$ or $\mathbf{Y}$.

Recently, molecular iodine has attracted great attention as a catalyst for organic transformations because it is inexpensive, readily available, nonmetallic, and environmentally-benign as compared to transition metal catalysts. Further, molecular iodine has received importance in organic synthesis due to its mild Lewis acidic character. Molecular iodine has also been used in the synthesis of various heterocyclic compounds, protection and deprotection of functional groups such as alcohols and carboxylic acids, amines, phenols, iodination reactions and oxidation reactions. ${ }^{11}$ Particularly, iodine has

\footnotetext{
${ }^{*}$ For correspondence
} 


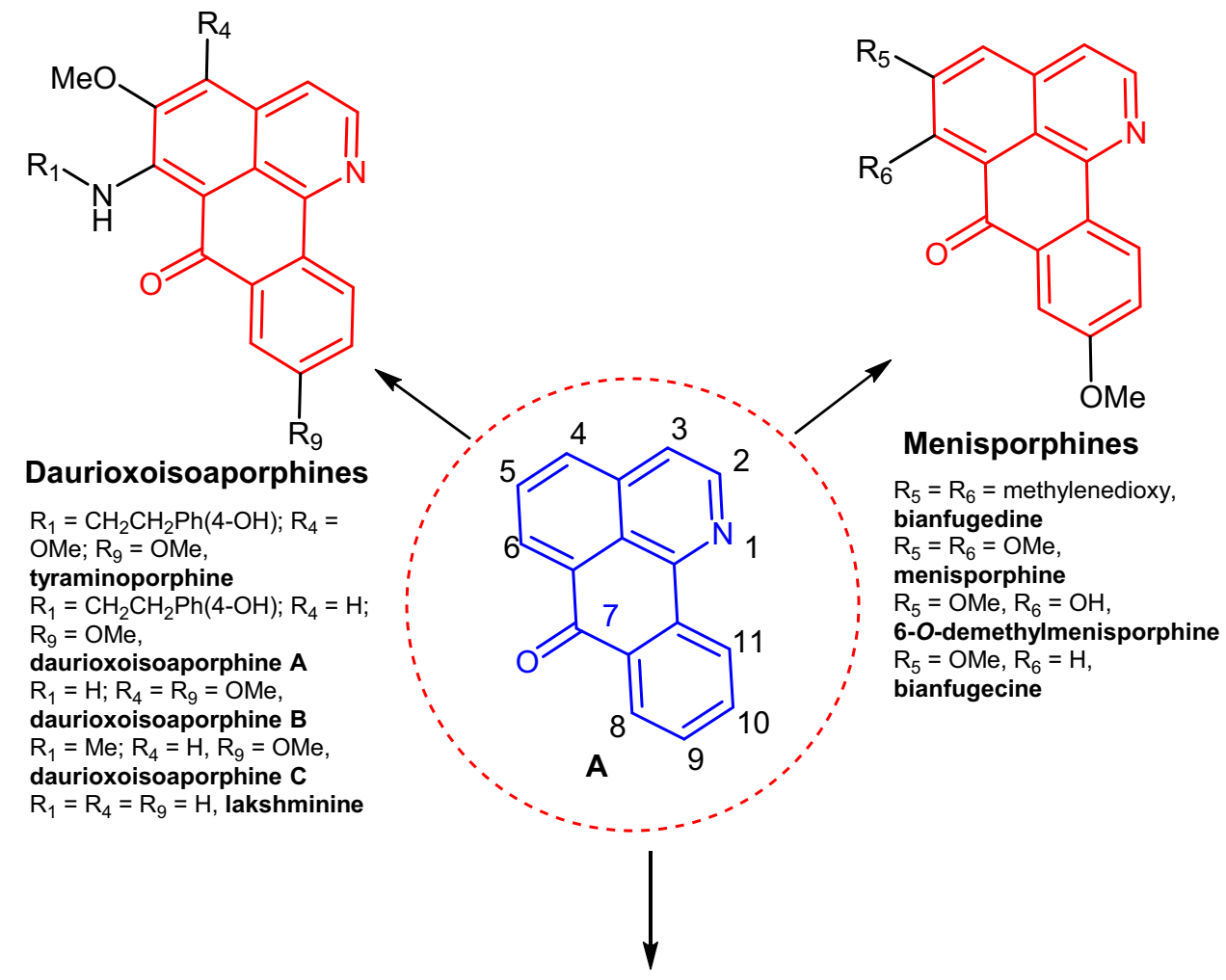<smiles></smiles>

1-Azabenzanthrones

Figure 1. Structure of oxoisoaporphine, azabenzanthrones, menisporphine alkaloids.<smiles>[X]c1cccc(C(=O)O)c1C1=NCCc2cc[R17]cc2C1=CCCC</smiles>

Figure 2. Synthetic routes to prepare 1-azabenzanthrone alkaloids.

been utilized for the oxidative aromatization of $\alpha, \beta$ unsaturated cyclic compounds. ${ }^{12}$ Similarly, oxidative aromatization of tetrahydroisoquinoline was achieved using $\mathrm{KI}$ and ${ }^{t} \mathrm{BuOOH}$ combination. ${ }^{13}$ To the best of our knowledge, direct utility of molecular iodine for oxidative dehydrogenation of tetrahydroisoindoloisoquinolinone system has not been reported.
Hence, herein, we report an oxidative dehydrogenation and ring opening of amide group in isoindoloisoquinolinones with molecular iodine for the first time to prepare $o$-isoquinolin-2-yl benzoic acids, the potential precursors for the synthesis of oxoisoaporphine alkaloids.

A simple and efficient methodology has been developed to prepare THIQs and THBCs through activation of imide carbonyl group using Lewis acid/Brønsted acid. ${ }^{14}$ Following this methodology, we synthesized the required starting materials in a single step from substituted phenethylphthalimides I for this studies. From the acquired knowledge from the synthesis of isoindoloisoquinolinones, one can visualize the precursor $\mathbf{X}$ from imide $\mathbf{I}$ via a $\mathrm{C}-\mathrm{N}$ and $\mathrm{C}-\mathrm{C}$ bond disconnections (Scheme 1). 


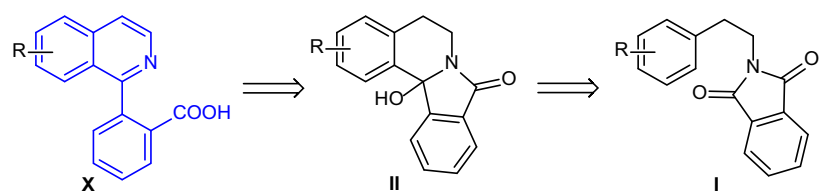

Scheme 1. Retrosynthetic analysis of isoquinolinylbenzoic acid $\mathbf{X}$.

\section{Experimental}

\subsection{General information}

Melting points reported in this paper are uncorrected and were determined using BUCHI M-560, BuchiLabortechnik AG, Switzerland. Infrared spectra were recorded on Thermo Nicolet iS10 FT-IR Spectrophotometer and are reported in frequency of absorption $\left(\mathrm{cm}^{-1}\right)$. Mass spectra were measured with Agilent-6530 B Q-TOF (ESI-HRMS). ${ }^{1} \mathrm{H}$ and ${ }^{13} \mathrm{C}$ NMR were recorded on Brucker AVANCE 400 spectrometer. NMR spectra for all the samples were measured in $\mathrm{CDCl}_{3}$ or DMSO- $d_{6}$ using TMS as an internal standard. The chemical shifts are expressed in $\delta \mathrm{ppm}$ down field from the signal of internal TMS. All solvents used in the reactions and chromatography were distilled and/or dried properly for purity using standard procedures. ${ }^{16}$ Dry dichloroethane was obtained by distillation over calcium hydride. Column chromatography was performed on Merck silica gel 100-200 mesh and TLC analysis was facilitated using phosphomolybdic acid and $\mathrm{KMnO}_{4}$ stain in addition to UV light with Merck $60 \mathrm{~F} 254$ pre-coated silica plates. Starting materials were prepared from the corresponding substituted phenethylphthalimides following the existing triflic acid-mediated imide carbonyl group activation methodology. ${ }^{14}$

\subsection{General procedure for the synthesis of ether derivatives (3a-3c, $\mathbf{3 e}-\mathbf{3 h}, \mathbf{3 j})$}

A $20 \mathrm{~mL}$-sealed tube was charged with hydroxy lactam $\mathbf{1}(0.4$ $\mathrm{mmol})$ and iodine $(0.4 \mathrm{mmol})$ in dry methanol $(2 \mathrm{~mL})$. The tube was flushed with nitrogen gas and closed with Teflon cap. The solution was heated at $100^{\circ} \mathrm{C}$ for $12 \mathrm{~h}$. The resulting suspension was cooled to room temperature and the solvent was removed under vacuum on rotary evaporator to dryness. Ethyl acetate $(5 \mathrm{~mL})$ was added with vigorous stirring and excess iodine was quenched by aqueous saturated $\mathrm{Na}_{2} \mathrm{~S}_{2} \mathrm{O}_{3}$ solution $(5 \mathrm{~mL})$. The organic layer was separated and aqueous layer was extracted with ethyl acetate $(2 \times 5 \mathrm{~mL})$. The combined organic extract was washed with brine solution and dried over anhydrous $\mathrm{Na}_{2} \mathrm{SO}_{4}$. Filtered and the solvent was removed under vacuum on rotary evaporator to dryness. The dried compound was purified through short silica gel column chromatography using hexane and ethyl acetate (90:10) as eluent to give desired products $(\mathbf{3 a}-\mathbf{3 c}, \mathbf{3 e}-\mathbf{3 h}, \mathbf{3 j})$. 2.2a 12b-Methoxy-5,12b-dihydroisoindolo[1,2-a]isoquinolin-8(6H)-one (3a): $97 \mathrm{mg}$, colorless solid, 92\% yield, M.p. $127-128^{\circ} \mathrm{C}$, IR $\left(\mathrm{KBr}, \mathrm{cm}^{-1}\right)$ : 3065, 2935, 2829 , 1707, 1608, 1458, 1301, 1103, 1064, 761; ${ }^{1} \mathrm{H}$ NMR (400 $\left.\mathrm{MHz}, \mathrm{CDCl}_{3}\right): \delta 7.92(\mathrm{~d}, J=7.6 \mathrm{~Hz}, 1 \mathrm{H}), 7.85(\mathrm{~d}, J=7.2$ $\mathrm{Hz}, 2 \mathrm{H}), 7.64(\mathrm{t}, J=7.6 \mathrm{~Hz}, 1 \mathrm{H}), 7.52(\mathrm{t}, J=7.6 \mathrm{~Hz}$, $1 \mathrm{H}), 7.30-7.23(\mathrm{~m}, 2 \mathrm{H}), 7.16(\mathrm{~d}, J=6.8 \mathrm{~Hz}, 1 \mathrm{H}), 4.43$ (ddd, $J=12.8,6.4,2.4 \mathrm{~Hz}, 1 \mathrm{H}$ ), 3.43 (ddd, $J=16.0,11.2$, $4.8 \mathrm{~Hz}, 1 \mathrm{H}$ ), 3.07 (ddd, $J=16.4,11.2,6.4 \mathrm{~Hz}, 1 \mathrm{H}), 2.99$ (s, 3H), 2.90-2.85 (m, 1H); $\left.{ }^{13} \mathrm{C} \mathrm{NMR} \mathrm{(100} \mathrm{MHz,} \mathrm{CDCl}_{3}\right): \delta$ 167.8, 144.1, 135.9, 134.9, 132.5, 132.4, 129.9, 129.4, 128.7, $127.5,126.8,123.8,123.7,90.4,50.5,35.2$, 29.1; HRMS-ESI $(\mathrm{m} / \mathrm{z}):[\mathrm{M}+\mathrm{H}]^{+}$Found 266.1182 and calculated 266.1181 for $\mathrm{C}_{17} \mathrm{H}_{16} \mathrm{NO}_{2}$.

\section{$2.2 \mathrm{~b}$ 3-Bromo-12b-methoxy-5,12b-dihydroisoindolo}

[1,2-a]isoquinolin-8(6H)-one $(3 \mathrm{~b})$ : $128 \mathrm{mg}$, colorless solid, $93 \%$ yield, M.p. $139-140^{\circ} \mathrm{C}$, IR $\left(\mathrm{KBr}, \mathrm{cm}^{-1}\right)$ : 2928 , 1707, 1610, 1440, 1378, 1109, 1014, 807, 761; ${ }^{1} \mathrm{H}$ NMR (400 $\left.\mathrm{MHz}, \mathrm{CDCl}_{3}\right): \delta 7.84(\mathrm{td}, J=7.6,0.8 \mathrm{~Hz}, 2 \mathrm{H}), 7.70(\mathrm{~d}$, $J=8.4 \mathrm{~Hz}, 1 \mathrm{H}), 7.63(\mathrm{td}, J=7.6,1.2 \mathrm{~Hz}, 1 \mathrm{H}), 7.51(\mathrm{td}$, $J=7.6,1.0 \mathrm{~Hz}, 1 \mathrm{H}), 7.38(\mathrm{dd}, J=8.4,2.0 \mathrm{~Hz}, 1 \mathrm{H}), 7.29(\mathrm{t}$, $J=1.2 \mathrm{~Hz}, 1 \mathrm{H}), 4.40(\mathrm{ddd}, J=8.8,6.3,2.6 \mathrm{~Hz}, 1 \mathrm{H}), 3.37$ (ddd, $J=13.2,11.2,4.6 \mathrm{~Hz}, 1 \mathrm{H}), 3.06-2.97(\mathrm{~m}, 1 \mathrm{H}), 2.96$ (s, 3H), 2.82 (ddd, $J=7.2,4.4,2.8 \mathrm{~Hz}, 1 \mathrm{H}) ;{ }^{13} \mathrm{C}$ NMR $(100$ $\left.\mathrm{MHz}, \mathrm{CDCl}_{3}\right): \delta$ 167.6, 143.6, 137.1, 134.9, 132.5, 132.2, 132.1, 130.1, 129.9, 129.2, 123.9, 123.5, 122.6, 90.1, 50.5, 34.7, 28.8; HRMS-ESI (m/z): $[\mathrm{M}+\mathrm{H}]^{+}$Found 344.0308 and calculated 344.0286 for $\mathrm{C}_{17} \mathrm{H}_{15} \mathrm{BrNO}_{2}$.

\section{2c 2,3,12b-Trimethoxy-5,12b-dihydroisoindolo[1,}

2-a]isoquinolin-8(6H)-one (3c): $103 \mathrm{mg}$, yellow semi solid, 76\% yield, IR $\left(\mathrm{KBr}, \mathrm{cm}^{-1}\right)$ : 2996, 2935, 2837 1704, $1612,1459,1061,1025,758 ;{ }^{1} \mathrm{H}$ NMR $\left(400 \mathrm{MHz}, \mathrm{CDCl}_{3}\right): \delta$ $7.87(\mathrm{~d}, J=7.6 \mathrm{~Hz}, 1 \mathrm{H}), 7.84(\mathrm{~d}, J=7.6 \mathrm{~Hz}, 1 \mathrm{H}), 7.64(\mathrm{td}$, $J=7.6,1.2 \mathrm{~Hz}, 1 \mathrm{H}), 7.53-7.49(\mathrm{~m}, 1 \mathrm{H}), 7.33(\mathrm{~s}, 1 \mathrm{H}), 6.59(\mathrm{~s}$, $1 \mathrm{H}), 4.45$ (ddd, $J=12.8,6.0,1.6 \mathrm{~Hz}, 1 \mathrm{H}), 3.94(\mathrm{~s}, 3 \mathrm{H}), 3.83$ (s, 3H), 3.67 (td, $J=12.8,4.4,1 \mathrm{H}), 3.05-2.98(\mathrm{~m}, 1 \mathrm{H}), 2.97$ (s, 3H), 2.75-2.69 (m, 1H); $\left.{ }^{13} \mathrm{C} \mathrm{NMR} \mathrm{(100} \mathrm{MHz,} \mathrm{CDCl}_{3}\right): \delta$ 167.8, 149.4, 147.9, 144.4, 132.4, 132.3, 129.8, 127.6, 127.6, 123.9, 123.3, 111.5, 110.3, 90.2, 56.2, 56.0, 50.5, 35.1, 28.8; HRMS-ESI $(\mathrm{m} / \mathrm{z}):[\mathrm{M}+\mathrm{H}]^{+}$Found 326.1376 and calculated 326.1392 for $\mathrm{C}_{19} \mathrm{H}_{20} \mathrm{NO}_{4}$.

\section{$2.2 \mathrm{~d}$ 1,4,12b-Trimethoxy-5,12b-dihydroisoindolo[1,}

2-a]isoquinolin-8(6H)-one (3e): $95 \mathrm{mg}$, red semi solid, $70 \%$ yield, IR (KBr, cm $\left.{ }^{-1}\right)$ : 3084, 2945, 2833, 1701, 1602, 1475, 1262, 1070, 985, 754; ${ }^{1} \mathrm{H}$ NMR (400 MHz, $\left.\mathrm{CDCl}_{3}\right)$ : $\delta 8.28(\mathrm{~d}, J=7.6 \mathrm{~Hz}, 1 \mathrm{H}), 7.83-7.80(\mathrm{~m}, 1 \mathrm{H}), 7.55(\mathrm{td}$, $J=7.6,1.2 \mathrm{~Hz}, 1 \mathrm{H}), 7.48(\mathrm{td}, J=7.2 .0 .8 \mathrm{~Hz}, 1 \mathrm{H})$, $6.81(\mathrm{~d}, J=8.8 \mathrm{~Hz}, 1 \mathrm{H}), 6.76(\mathrm{~d}, J=8.8 \mathrm{~Hz}, 1 \mathrm{H}), 4.47$ (ddd, $J=7.6,6.4,1.2 \mathrm{~Hz}, 1 \mathrm{H}), 3.96(\mathrm{~s}, 3 \mathrm{H}), 3.74(\mathrm{~s}, 3 \mathrm{H})$, 3.27-3.19 (m, 1H), 2.99 (s, 3H), 2.94-2.88 (m, 1H), 2.80$2.70(\mathrm{~m}, 1 \mathrm{H}) ;{ }^{13} \mathrm{C}$ NMR $\left(100 \mathrm{MHz}, \mathrm{CDCl}_{3}\right): \delta 167.6,152.4$, 151.6, 144.0, 132.8, 131.8, 129.8, 126.8, 126.7, 125.6, 123.2, $110.7,110.4,91.9,56.1,55.9,50.3,33.8,24.1$; HRMS-ESI 
$(\mathrm{m} / \mathrm{z}):[\mathrm{M}+\mathrm{H}]^{+}$Found 326.1386 and calculated 326.1392 for $\mathrm{C}_{19} \mathrm{H}_{20} \mathrm{NO}_{4}$.

2.2e 2,3,4,12b-Tetramethoxy-5,12b-dihydroisoindolo [1,2-a]isoquinolin-8(6H)-one (3f): $94 \mathrm{mg}$, yellow semi solid, $66 \%$ yield, IR $\left(\mathrm{KBr}, \mathrm{cm}^{-1}\right): 2941,2836,1702,1606$, 1459, 1112, 1040, 747; ${ }^{1} \mathrm{H}$ NMR (400 MHz, $\left.\mathrm{CDCl}_{3}\right): \delta 7.85$ $(\mathrm{dd}, J=13.2,7.6 \mathrm{~Hz}, 2 \mathrm{H}), 7.63(\mathrm{td}, J=7.6,0.8 \mathrm{~Hz}$, $1 \mathrm{H}), 7.51$ (td, $J=7.6,0.8 \mathrm{~Hz}, 1 \mathrm{H}), 7.18$ (s, 1H), 4.45 (ddd, $J=8.0,6.0,2.0 \mathrm{~Hz}, 1 \mathrm{H}), 3.92(\mathrm{~s}, 3 \mathrm{H}), 3.81(\mathrm{~s}, 3 \mathrm{H}), 3.79$ (s, 3H), 3.32-3.25 (m, 1H), $2.96(\mathrm{~s}, 3 \mathrm{H}), 2.93-2.87(\mathrm{~m}, 1 \mathrm{H})$, 2.84-2.71 (m, 1H); ${ }^{13} \mathrm{C}$ NMR (100 MHz, $\left.\mathrm{CDCl}_{3}\right): \delta 167.5$, 152.2, 151.1, 144.1, 142.5, 132.4, 132.3, 130.9, 129.9, 123.9, 123.4, 122.0, 106.6, 90.1, 60.8, 60.6, 56.2, 50.4, 34.6, 22.9; HRMS-ESI $(\mathrm{m} / \mathrm{z}):[\mathrm{M}+\mathrm{H}]^{+}$Found 356.1501 and calculated 356.1498 for $\mathrm{C}_{20} \mathrm{H}_{22} \mathrm{NO}_{5}$.

\section{2f 12b-Methoxy-3-methyl-5,12b-dihydroisoindolo}

[1,2-a]isoquinolin-8(6H)-one (3g): $75 \mathrm{mg}$, colorless solid, $67 \%$ yield, M.p. $134-137^{\circ} \mathrm{C}$, IR $\left(\mathrm{KBr}, \mathrm{cm}^{-1}\right)$ : 2930 , 2817, 1702, 1611, 1457, 1380, 1102, 1057, 755; ${ }^{1} \mathrm{H}$ NMR $\left(400 \mathrm{MHz}, \mathrm{CDCl}_{3}\right): \delta 7.91(\mathrm{~d}, J=7.6 \mathrm{~Hz}, 1 \mathrm{H}), 7.83(\mathrm{dt}$, $J=7.6,0.8 \mathrm{~Hz}, 1 \mathrm{H}), 7.73(\mathrm{~d}, J=8.0 \mathrm{~Hz}, 1 \mathrm{H}), 7.63(\mathrm{td}$, $J=7.6,1.2 \mathrm{~Hz}, 1 \mathrm{H}), 7.50(\mathrm{td}, J=7.2,0.8 \mathrm{~Hz}, 1 \mathrm{H}), 7.09$ $(\mathrm{d}, J=8.0 \mathrm{~Hz}, 1 \mathrm{H}), 6.97$ (s, 1H), 4.41 (ddd, $J=12.8,6.0$, $1.8 \mathrm{~Hz}, 1 \mathrm{H}), 3.40$ (ddd, $J=16.0,11.2,4.4 \mathrm{~Hz}, 1 \mathrm{H}), 3.07-$ $2.98(\mathrm{~m}, 1 \mathrm{H}), 2.97$ (s, 3H), 2.82 (ddd, $J=16.0,4.4,2.4 \mathrm{~Hz}$, $1 \mathrm{H}), 2.29$ (s, 3H); ${ }^{13} \mathrm{C}$ NMR (100 MHz, $\left.\mathrm{CDCl}_{3}\right): \delta 167.8$, 144.2, 138.6, 134.7, 133.0, 132.4, 132.3, 129.9, 129.8, 127.7, 127.4, 123.8, 123.6, 90.4, 50.5, 35.1, 29.1, 21.1; HRMS-ESI $(\mathrm{m} / \mathrm{z}):[\mathrm{M}+\mathrm{H}]^{+}$Found 280.1371 and calculated 280.1338 for $\mathrm{C}_{18} \mathrm{H}_{18} \mathrm{NO}_{2}$.

\section{$2.2 \mathrm{~g}$ 11,12b-Dimethoxy-5,12b-dihydroisoindolo [1,2} -a]isoquinolin-8(6H)-one (3h): $46 \mathrm{mg}$, colorless solid, $39 \%$ yield, M.p. $159-161^{\circ} \mathrm{C}$, IR $\left(\mathrm{KBr}, \mathrm{cm}^{-1}\right): 2934,2834$, 1699, 1610, 1449, 1105, 1062, 765; ${ }^{1} \mathrm{H}$ NMR (400 MHz, $\left.\mathrm{CDCl}_{3}\right): \delta 7.82(\mathrm{dd}, J=8.0,2.0 \mathrm{~Hz}, 1 \mathrm{H}), 7.76(\mathrm{~d}, J=8.4$ $\mathrm{Hz}, 1 \mathrm{H}), 7.39(\mathrm{~d}, J=2.0 \mathrm{~Hz}, 1 \mathrm{H}), 7.30-7.22(\mathrm{~m}, 2 \mathrm{H}), 7.16$ $(\mathrm{d}, J=6.8 \mathrm{~Hz}, 1 \mathrm{H}), 7.08(\mathrm{dd}, J=8.4,2.0 \mathrm{~Hz}, 1 \mathrm{H}) 4.39$ (ddd, $J=8.8,6.0,2.4 \mathrm{~Hz}, 1 \mathrm{H}), 3.93(\mathrm{~s}, 3 \mathrm{H}), 3.40(\mathrm{ddd}$, $J=12.4,11.2,4.8 \mathrm{~Hz}, 1 \mathrm{H}), 3.09-3.03(\mathrm{~m} \mathrm{1H}), 3.01(\mathrm{~s}, 3 \mathrm{H})$, 2.85 (ddd, $J=11.2,4.4,3.2 \mathrm{~Hz}, 1 \mathrm{H}) ;{ }^{13} \mathrm{C}$ NMR $(100 \mathrm{MHz}$, $\left.\mathrm{CDCl}_{3}\right): \delta 167.9,163.4,146.3,135.9,135.1,129.4,128.6$, 127.3, 126.8, 125.2, 125.0, 115.5, 109.4, 90.1, 55.9, 50.5, 35.3, 29.0; HRMS-ESI (m/z): $[\mathrm{M}+\mathrm{H}]^{+}$Found 296.1277 and calculated 296.1287 for $\mathrm{C}_{18} \mathrm{H}_{18} \mathrm{NO}_{3}$.

2.2h 12b-Methoxy-5,12b-dihydro-[1,3]dioxolo[4,5-g] isoindolo[1,2-a]isoquinolin-8(6H)-one (3j): $83 \mathrm{mg}$, light yellow semi solid, $67 \%$ yield, IR $\left(\mathrm{KBr}, \mathrm{cm}^{-1}\right)$ : 2935, 2893, 1703, 1621, 1480, 1039, 760; ${ }^{1} \mathrm{H}$ NMR (400 MHz, $\left.\mathrm{CDCl}_{3}\right): \delta 7.85(\mathrm{dd}, J=7.6,3.6 \mathrm{~Hz}, 2 \mathrm{H}), 7.64(\mathrm{td}, J=7.6$, $0.8 \mathrm{~Hz}, 1 \mathrm{H}), 7.52(\mathrm{t}, J=7.6 \mathrm{~Hz}, 1 \mathrm{H}), 7.31(\mathrm{~s}, 1 \mathrm{H}), 6.58(\mathrm{~s}$, $1 \mathrm{H}), 5.91$ (dd, $J=13.6,1.2 \mathrm{~Hz}, 2 \mathrm{H}), 4.40$ (ddd, $J=8.4,6.0$, $2.4 \mathrm{~Hz}, 1 \mathrm{H}), 3.36(\mathrm{ddd}, J=12.8,11.6,4.4 \mathrm{~Hz}, 1 \mathrm{H}), 3.01-2.93$ (m 1H), $2.96(\mathrm{~s}, 3 \mathrm{H}), 2.73(\mathrm{ddd}, J=6.8,4.4,2.4 \mathrm{~Hz}, 1 \mathrm{H}) ;{ }^{13} \mathrm{C}$
NMR (100 MHz, $\left.\mathrm{CDCl}_{3}\right): \delta 167.8,147.9,146.7,144.2,132.4$, 132.3, 129.9, 129.0, 128.9, 123.9, 123.5, 108.9, 107.4, 101.3, 90.4, 50.5, 35.1, 29.3; HRMS-ESI $(\mathrm{m} / \mathrm{z}):[\mathrm{M}+\mathrm{H}]^{+}$Found 310.1087 and calculated 310.1079 for $\mathrm{C}_{18} \mathrm{H}_{16} \mathrm{NO}_{3}$.

\subsection{General procedure for the synthesis of}

2-(isoquinolin-1-yl)benzoic acid/ ester derivatives $(2 a-2 i / 4 a, 4 c, 4 e, 4 h)$

A $20 \mathrm{~mL}$-sealed tube was charged with corresponding substrate 1 or $3(0.2 \mathrm{mmol})$ and iodine (1 equiv.) in dry 1,2-dichloroethane $(2 \mathrm{~mL})$. The tube was flushed with nitrogen gas and closed with Teflon cap. The solution was heated at $100^{\circ} \mathrm{C}$ for $12 \mathrm{~h}$. The resulting suspension was cooled to room temperature and ethyl acetate $(5 \mathrm{~mL})$ was added with vigorous stirring and stirring was continued for $1 \mathrm{~h}$. Excess iodine was quenched by saturated aqueous $\mathrm{Na}_{2} \mathrm{~S}_{2} \mathrm{O}_{3}$ solution $(5 \mathrm{~mL})$. The organic layer was separated and aqueous layer was extracted with ethyl acetate $(2 \times 5 \mathrm{~mL})$. The combined organic extract was washed with brine solution and dried over anhydrous $\mathrm{Na}_{2} \mathrm{SO}_{4}$. Filtered and the solvent was removed under vacuum on rotary evaporator to dryness. The dried compound was purified through short silica gel column chromatography using methanol as eluent to give desired product $\mathbf{2}$ and hexane and ethyl acetate (90:10) as eluent to give desired product 4 .

2.3a 2-(Isoquinolin-1-yl)benzoic acid (2a): $46 \mathrm{mg}$, colorless solid, $92 \%$ yield, M.p. $245-246^{\circ} \mathrm{C}$, IR $\left(\mathrm{KBr}, \mathrm{cm}^{-1}\right)$ : $3425,1685,1594,1385,1260,762 ;{ }^{1} \mathrm{H}$ NMR $(400 \mathrm{MHz}$, DMSO- $\left.d_{6}\right): \delta 8.48(\mathrm{~d}, J=6.0 \mathrm{~Hz}, 1 \mathrm{H}), 8.01-7.98(\mathrm{~m}$, $2 \mathrm{H}), 7.80(\mathrm{~d}, J=5.6 \mathrm{~Hz}, 1 \mathrm{H}), 7.76-7.72(\mathrm{~m}, 1 \mathrm{H}), 7.68$ $(\operatorname{td}, J=7.6,1.6 \mathrm{~Hz}, 1 \mathrm{H}), 7.61(\mathrm{td}, J=7.6,1.6 \mathrm{~Hz}, 1 \mathrm{H})$, $7.58-7.53(\mathrm{~m}, 2 \mathrm{H}), 7.42(\mathrm{dd}, J=7.6,1.2 \mathrm{~Hz}, 1 \mathrm{H}) ;{ }^{13} \mathrm{C} \mathrm{NMR}$ $\left(100 \mathrm{MHz}, \mathrm{DMSO}-d_{6}\right): \delta 167.8,160.7,141.6,139.9,135.5$, 132.1, 131.4, 130.7, 130.0, 129.8, 128.4, 127.4, 126.9, 126.7, 126.3, 119.7; HRMS-ESI (m/z): $[\mathrm{M}+\mathrm{H}]^{+}$Found 250.0862 and calculated 250.0868 for $\mathrm{C}_{16} \mathrm{H}_{12} \mathrm{NO}_{2}$.

\section{3b 2-(6-Bromoisoquinolin-1-yl)benzoic acid $(2 \boldsymbol{b})$ :}

$41 \mathrm{mg}$, colorless solid, $62 \%$ yield, M.p. $278-281^{\circ} \mathrm{C}$, IR $(\mathrm{KBr}$, $\left.\mathrm{cm}^{-1}\right)$ : 3401, 2831, 2725, 1706, 1594, 1375, 1119, 770; ${ }^{1} \mathrm{H}$ NMR (400 MHz, DMSO- $\left.d_{6}\right): \delta 8.53(\mathrm{~d}, J=5.6 \mathrm{~Hz}, 1 \mathrm{H})$, $8.17-8.16(\mathrm{~m}, 1 \mathrm{H}), 8.01(\mathrm{dd}, J=8.0,1.6 \mathrm{~Hz}, 1 \mathrm{H}), 7.81$ $-7.79(\mathrm{~m}, 1 \mathrm{H}), 7.72(\mathrm{td}, J=7.6,1.6 \mathrm{~Hz}, 1 \mathrm{H}), 7.65(\mathrm{td}$, $J=7.6,1.6 \mathrm{~Hz}, 1 \mathrm{H}), 7.57(\mathrm{~d}, J=1.6 \mathrm{~Hz}, 2 \mathrm{H}), 7.45(\mathrm{dd}$, $J=7.6,1.2 \mathrm{~Hz}, 1 \mathrm{H}) ;{ }^{13} \mathrm{C}$ NMR $\left(100 \mathrm{MHz}, \mathrm{DMSO}-d_{6}\right): \delta$ $167.7,160.9,142.8,139.5,136.4,134.8,132.0,131.5,130.7$, 129.9, 128.8, 128.7, 128.0, 125.6, 125.2, 119.0; HRMS-ESI $(\mathrm{m} / \mathrm{z}):[\mathrm{M}+\mathrm{H}]^{+}$Found 327.9974 and calculated 327.9973 for $\mathrm{C}_{16} \mathrm{H}_{11} \mathrm{BrNO}_{2}$.

2.3c 2-(6,7-Dimethoxyisoquinolin-1-yl)benzoic acid (2c): $46 \mathrm{mg}$, colorless solid, $75 \%$ yield, M.p. $220-221^{\circ} \mathrm{C}$, IR $\left(\mathrm{KBr}, \mathrm{cm}^{-1}\right)$ : 3450, 2925, 2834, 1689, 1565, 1481, 1417, $1384,1278,772 ;{ }^{1} \mathrm{H}$ NMR (400 MHz, DMSO- $\left.d_{6}\right): \delta 8.24$ (d, $J=6.0 \mathrm{~Hz}, 1 \mathrm{H}), 7.88(\mathrm{dd}, J=5.6,3.2 \mathrm{~Hz}, 1 \mathrm{H}), 7.52(\mathrm{~d}$, $J=5.6 \mathrm{~Hz}, 1 \mathrm{H}), 7.42(\mathrm{dd}, J=5.2,3.2 \mathrm{~Hz}, 2 \mathrm{H}), 7.29(\mathrm{~s}$, 
$1 \mathrm{H}), 7.22(\mathrm{dd}, J=5.6,3.2 \mathrm{~Hz}, 1 \mathrm{H}), 6.95(\mathrm{~s}, 1 \mathrm{H}), 3.90(\mathrm{~s}$, $3 \mathrm{H}), 3.62$ (s, 3H); ${ }^{13} \mathrm{C}$ NMR (100 MHz, DMSO-d $): \delta 171.8$, $160.5,152.0,149.0,140.7,140.3,138.9,132.1,130.1,129.1$, 128.1, 127.3, 123.0, 117.9, 105.8, 105.0, 55.7, 55.2; HRMSESI (m/z): $[\mathrm{M}+\mathrm{H}]^{+}$Found 310.1073 and calculated 310.1079 for $\mathrm{C}_{18} \mathrm{H}_{16} \mathrm{NO}_{4}$.

2.3d 2-(6,8-Dimethoxyisoquinolin-1-yl)benzoic acid (2d): $40 \mathrm{mg}$, colorless solid, $65 \%$ yield, M.p. $198-200^{\circ} \mathrm{C}$, IR $\left(\mathrm{KBr}, \mathrm{cm}^{-1}\right): 3457,3008,2934,2854,1701,1668,1615$, 1586, 1413, 1158, 760; ${ }^{1} \mathrm{H}$ NMR (400 MHz, $\left.\mathrm{CDCl}_{3}\right): \delta 8.27$ $(\mathrm{d}, J=5.2 \mathrm{~Hz}, 1 \mathrm{H}), 7.99(\mathrm{~d}, J=7.2 \mathrm{~Hz}, 1 \mathrm{H}), 7.52-7.46$ (m, 2H), $7.42(\mathrm{t}, J=7.2 \mathrm{~Hz}, 1 \mathrm{H}), 7.19(\mathrm{~d}, J=7.6 \mathrm{~Hz}, 1 \mathrm{H})$, $6.71(\mathrm{~d}, J=2.4 \mathrm{~Hz}, 1 \mathrm{H}), 6.41(\mathrm{~d}, J=2.0 \mathrm{~Hz}, 1 \mathrm{H}), 3.93$ (s, 3H), 3.41 (s, 3H); $\left.{ }^{13} \mathrm{C} \mathrm{NMR} \mathrm{(100} \mathrm{MHz,} \mathrm{CDCl}_{3}\right)$ : 162.7, 159.4, 158.6, 152.7, 140.3, 132.3, 130.4, 130.1, 130.0, 129.3, $127.4,124.3$, 119.5, 115.9, 100.2, 97.8, 55.7, 55.6; HRMSESI $(\mathrm{m} / \mathrm{z}):[\mathrm{M}+\mathrm{H}]^{+}$Found 310.1075 and calculated 310.1079 for $\mathrm{C}_{18} \mathrm{H}_{16} \mathrm{NO}_{4}$.

2.3e 2-(5,8-Dimethoxyisoquinolin-1-yl)benzoic acid (2e): $28 \mathrm{mg}$, yellow solid, $45 \%$ yield, M.p. $211-213^{\circ} \mathrm{C}$, IR $\left(\mathrm{KBr}, \mathrm{cm}^{-1}\right): 3449,3005,2940,2843,1671,1577,1417$, $1384,1122,768 ;{ }^{1} \mathrm{H}$ NMR (400 MHz, DMSO- $\left.d_{6}\right): \delta 8.45(\mathrm{~d}$, $J=5.6 \mathrm{~Hz}, 1 \mathrm{H}), 7.94-7.89(\mathrm{~m}, 2 \mathrm{H}), 7.56(\mathrm{td}, J=7.2,1.2 \mathrm{~Hz}$, $1 \mathrm{H}), 7.47(\mathrm{td}, J=7.2,1.6 \mathrm{~Hz}, 1 \mathrm{H}), 7.19(\mathrm{dd}, J=7.2,0.8$ $\mathrm{Hz}, 1 \mathrm{H}), 7.10(\mathrm{~d}, J=8.8 \mathrm{~Hz}, 1 \mathrm{H}), 6.87(\mathrm{~d}, J=8.8 \mathrm{~Hz}, 1 \mathrm{H})$, $3.95(\mathrm{~s}, 3 \mathrm{H}), 3.34(\mathrm{~s}, 3 \mathrm{H}) ;{ }^{13} \mathrm{C} \mathrm{NMR}\left(100 \mathrm{MHz}, \mathrm{DMSO}-d_{6}\right): \delta$ $167.7,149.8,147.8,145.8,141.5,137.2$, 130.6, 130.0, 129.5, 129.0, 128.7, 126.8, 119.3, 113.1, 108.3, 107.0, 56.0, 55.7; HRMS-ESI $(\mathrm{m} / \mathrm{z}):[\mathrm{M}+\mathrm{H}]^{+}$Found 310.1089 and calculated 310.1079 for $\mathrm{C}_{18} \mathrm{H}_{16} \mathrm{NO}_{4}$.

2.3f 2-(5,6,7-Trimethoxyisoquinolin-1-yl)benzoic acid (2f): $32 \mathrm{mg}$, colorless semi solid, 51\% yield, IR ( $\mathrm{KBr}$, $\left.\mathrm{cm}^{-1}\right): 3425,3002,2945,2851,1682,1586,1510,1420$, 1160,$670 ;{ }^{1} \mathrm{H}$ NMR (400 MHz, DMSO- $\left.d_{6}\right): \delta 8.36-8.28(\mathrm{~m}$, $1 \mathrm{H}), 7.89-7.85(\mathrm{~m}, 1 \mathrm{H}), 7.50-7.40(\mathrm{~m}, 3 \mathrm{H}), 7.19(\mathrm{t}, J=2.4$ $\mathrm{Hz}, 1 \mathrm{H}), 6.83-6.71(\mathrm{~m}, 1 \mathrm{H}), 3.96$ (s, 3H), 3.87 (s, 3H), 3.84 $(\mathrm{s}, 3 \mathrm{H})$; HRMS-ESI $(\mathrm{m} / \mathrm{z}):[\mathrm{M}+\mathrm{H}]^{+}$Found 340.1186 and caluclated 340.1185 for $\mathrm{C}_{19} \mathrm{H}_{18} \mathrm{NO}_{5}$.

\section{$2.3 \mathrm{~g}$ 2-(6-Methylisoquinolin-1-yl)benzoic acid (2g):}

$28 \mathrm{mg}$, colorless solid, 53\% yield, M.p. $232-233^{\circ} \mathrm{C}$, IR $(\mathrm{KBr}$, $\left.\mathrm{cm}^{-1}\right): 3416,2925,2850,1682,1589,1468,1385,704 ;{ }^{1} \mathrm{H}$ NMR (400 MHz, DMSO-d 6 ): $\delta$ 8.50-8.25 (m, 2H), 8.07$7.91(\mathrm{~m}, 1 \mathrm{H}), 7.66-7.56(\mathrm{~m}, 1 \mathrm{H}), 7.44-7.36$ (m, 3H), 7.156.98 (m, 1H), 6.89-6.49 (m, 1H), 1.77 (s, 3H); HRMS-ESI $(\mathrm{m} / \mathrm{z}):[\mathrm{M}+\mathrm{H}]^{+}$Found 264.1028 and calculated 264.1025 for $\mathrm{C}_{17} \mathrm{H}_{14} \mathrm{NO}_{2}$.

2.3h 2-(Isoquinolin-1-yl)-4-methoxybenzoic acid (2h): $42 \mathrm{mg}$, colorless semi solid, $76 \%$ yield, IR $\left(\mathrm{KBr}, \mathrm{cm}^{-1}\right): 3399$, 3058, 2934, 1696, 1603, 1451, 1233, 1027, 754; ${ }^{1} \mathrm{H}$ NMR (400 MHz, DMSO- $d_{6}$ ): $\delta 8.47(\mathrm{~d}, J=6.0 \mathrm{~Hz}, 1 \mathrm{H}), 8.01$ $(\mathrm{t}, J=8.8 \mathrm{~Hz}, 2 \mathrm{H}), 7.80(\mathrm{~d}, J=5.6 \mathrm{~Hz}, 1 \mathrm{H}), 7.75-7.71$ (m, 1H), 7.54-7.53 (m, 2H), $7.17(\mathrm{dd}, J=8.8,2.4 \mathrm{~Hz}, 1 \mathrm{H})$, $6.90(\mathrm{~d}, J=2.4 \mathrm{~Hz}, 1 \mathrm{H}), 3.83(\mathrm{~s}, 3 \mathrm{H}) ;{ }^{13} \mathrm{C} \mathrm{NMR}(100 \mathrm{MHz}$, DMSO- $\left.d_{6}\right): \delta 167.1,161.7,160.9,142.6,141.6,135.5,132.4$, 130.1, 127.5, 126.9, 126.4, 123.6, 119.9, 116.1, 113.9, 55.7; HRMS-ESI $(\mathrm{m} / \mathrm{z}):[\mathrm{M}+\mathrm{H}]^{+}$Found 280.0957 and calculated 280.0974 for $\mathrm{C}_{17} \mathrm{H}_{14} \mathrm{NO}_{3}$.

$2.3 \mathrm{i}$ 2-(Benzo[g]isoquinolin-1-yl)benzoic acid (2i): $25 \mathrm{mg}$, red solid, $41 \%$ yield, M.p. $244-245^{\circ} \mathrm{C}$, IR $(\mathrm{KBr}$, $\left.\mathrm{cm}^{-1}\right): 3425,2846,2781,1722,1600,1460,1290,813 ;{ }^{1} \mathrm{H}$ NMR (400 MHz, DMSO-d $\left.d_{6}\right): \delta 8.52(\mathrm{~d}, J=5.2 \mathrm{~Hz}, 1 \mathrm{H}), 8.05$ - $8.01(\mathrm{~m}, 2 \mathrm{H}), 7.94(\mathrm{~d}, J=8.0 \mathrm{~Hz}, 1 \mathrm{H}), 7.85(\mathrm{~d}, J=8.8$ $\mathrm{Hz}, 1 \mathrm{H}), 7.79(\mathrm{~d}, J=5.2 \mathrm{~Hz}, 1 \mathrm{H}), 7.60(\mathrm{~d}, J=8.8 \mathrm{~Hz}$, $1 \mathrm{H}), 7.54-7.42(\mathrm{~m}, 3 \mathrm{H}), 7.17-7.13(\mathrm{~m}, 1 \mathrm{H}) 6.91(\mathrm{~d}, J=7.2$ $\mathrm{Hz}, 1 \mathrm{H}) ;{ }^{13} \mathrm{C}$ NMR $\left(100 \mathrm{MHz}, \mathrm{DMSO}-d_{6}\right): \delta 170.4,160.9$, $144.7,143.2$, 137.6, 136.3, 133.8, 133.4, 132.1, 130.9, 130.8, 129.7, 129.3, 128.2, 127.5, 127.1, 126.7, 126.1, 123.9, 120.6; HRMS-ESI (m/z): $[\mathrm{M}+\mathrm{H}]^{+}$Found 300.1026 and calculated 300.1025 for $\mathrm{C}_{20} \mathrm{H}_{14} \mathrm{NO}_{2}$.

2.3j Methyl 2-(isoquinolin-1-yl)benzoate (4a): 28 $\mathrm{mg}$, colorless semi solid, $53 \%$ yield, IR $\left(\mathrm{KBr}, \mathrm{cm}^{-1}\right): 3056$, 2942, 2849, 1723, 1569, 1441, 1285, 1081, 760; ${ }^{1} \mathrm{H}$ NMR $\left(400 \mathrm{MHz}, \mathrm{CDCl}_{3}\right): \delta 8.57(\mathrm{~d}, J=5.6 \mathrm{~Hz}, 1 \mathrm{H}), 8.12(\mathrm{dd}$, $J=8.0,1.6 \mathrm{~Hz}, 1 \mathrm{H}), 7.88(\mathrm{~d}, J=8.4 \mathrm{~Hz}, 1 \mathrm{H}), 7.68-7.61(\mathrm{~m}$, $4 \mathrm{H}), 7.57(\mathrm{td}, J=7.6,1.2 \mathrm{~Hz}, 1 \mathrm{H}), 7.50(\mathrm{td}, J=7.6,1.2 \mathrm{~Hz}$, 1H), 7.46-7.44 (m, 1H), 3.41 (s, 3H); ${ }^{13} \mathrm{C} \mathrm{NMR} \mathrm{(100} \mathrm{MHz,}$ $\left.\mathrm{CDCl}_{3}\right): \delta 167.2,161.1,141.8,140.8,136.1,132.0,130.9$, 130.8, 130.5, 130.1, 128.6, 127.5, 127.3, 127.0, 126.8, 120.1, 51.9; HRMS-ESI (m/z): $[\mathrm{M}+\mathrm{Na}]^{+}$Found 286.0833 and calculated 286.0844 for $\mathrm{C}_{17} \mathrm{H}_{13} \mathrm{NNaO}_{2}$.

2.3k Methyl 2-(6,7-dimethoxyisoquinolin-1-yl)benzoate (4c): $27 \mathrm{mg}$, colorless semi solid, 42\% yield, IR (KBr, $\left.\mathrm{cm}^{-1}\right): 3017,2926,2853,1724,1617,1504,1425,1270$, 1127, 1027, 762; ${ }^{1} \mathrm{H}$ NMR (400 $\left.\mathrm{MHz} \mathrm{CDCl}_{3}\right): \delta 8.45(\mathrm{~d}$, $J=5.6 \mathrm{~Hz}, 1 \mathrm{H}), 8.08(\mathrm{dd}, J=7.6,1.2 \mathrm{~Hz}, 1 \mathrm{H}), 7.67(\mathrm{td}$, $J=7.6,1.2 \mathrm{~Hz}, 1 \mathrm{H}), 7.62-7.48(\mathrm{~m}, 3 \mathrm{H}), 7.13(\mathrm{~s}, 1 \mathrm{H}), 6.87$ (s, 1H), 4.04 (s, 3H), 3.77 (s, 3H), $3.44(\mathrm{~s}, 3 \mathrm{H}) ;{ }^{13} \mathrm{C}$ NMR $(100$ $\left.\mathrm{MHz} \mathrm{CDCl}_{3}\right): \delta 167.6,158.3,152.8,150.2,140.9,140.7$, 133.1, 132.1, 131.1, 130.9, 130.4, 128.6, 123.2, 119.0, 105.1, 104.8, 56.2, 55.9, 52.0; HRMS-ESI $(\mathrm{m} / \mathrm{z})$ : $[\mathrm{M}+\mathrm{H}]^{+}$Found 324.1233 and calculated 342.1236 for $\mathrm{C}_{19} \mathrm{H}_{18} \mathrm{NO}_{4}$.

\subsection{Methyl 2-(5,8-dimethoxyisoquinolin-1-yl)benzo-} ate (4e): $28 \mathrm{mg}$, colorless solid, 46\% yield, M.p. 159$160^{\circ} \mathrm{C}, \mathrm{IR}\left(\mathrm{KBr}, \mathrm{cm}^{-1}\right)$ : 3067, 2944, 2840, 1723, 1616, 1563, 1452, 1259, 1114, 778, 762; ${ }^{1} \mathrm{H}$ NMR (400 MHz, $\left.\mathrm{CDCl}_{3}\right): \delta$ $8.56(\mathrm{~d}, J=6.0 \mathrm{~Hz}, 1 \mathrm{H}), 8.06-8.02(\mathrm{~m}, 2 \mathrm{H}), 7.56(\mathrm{td}, J=7.2$, $1.2 \mathrm{~Hz}, 1 \mathrm{H}), 7.44(\mathrm{td}, J=7.6,1.2 \mathrm{~Hz}, 1 \mathrm{H}), 7.32(\mathrm{dd}, J=7.6$, $1.2 \mathrm{~Hz}, 1 \mathrm{H}), 6.90(\mathrm{~d}, J=8.4 \mathrm{~Hz}, 1 \mathrm{H}), 7.69$ (d, $J=8.4 \mathrm{~Hz}$, $1 \mathrm{H}), 3.98(\mathrm{~s}, 3 \mathrm{H}), 3.43(\mathrm{~s}, 3 \mathrm{H}), 3.40(\mathrm{~s}, 3 \mathrm{H}) ;{ }^{13} \mathrm{C}$ NMR $(100$ $\left.\mathrm{MHz}, \mathrm{CDCl}_{3}\right): \delta 167.3,158.6,150.3,148.7,146.1,141.8$, 131.4, 130.2, 129.5, 129.3, 129.1, 127.0, 120.1, 114.0, 107.7, 106.4, 56.0, 55.8, 51.7; HRMS-ESI $(\mathrm{m} / \mathrm{z}):[\mathrm{M}+\mathrm{H}]^{+}$Found 324.1233 and calculated 342.1236 for $\mathrm{C}_{19} \mathrm{H}_{18} \mathrm{NO}_{4}$. 
2.3m Methyl 2-(isoquinolin-1-yl)-4-methoxybenzoate (4h): $36 \mathrm{mg}$, colorless semi solid, $61 \%$ yield, IR (KBr, $\left.\mathrm{cm}^{-1}\right)$ : 2929, 2851, 1717, 1603, 1429, 1271, 1123, 1031, 828; ${ }^{1} \mathrm{H}$ NMR $\left(400 \mathrm{MHz}, \mathrm{CDCl}_{3}\right): \delta 8.49(\mathrm{~d}, J=5.6 \mathrm{~Hz}, 1 \mathrm{H})$, $8.05(\mathrm{~d}, J=8.8 \mathrm{~Hz}, 1 \mathrm{H}), 7.81(\mathrm{~d}, J=8.0 \mathrm{~Hz}, 1 \mathrm{H}), 7.61-$ $7.56(\mathrm{~m}, 2 \mathrm{H}), 7.54-7.51(\mathrm{~m}, 1 \mathrm{H}), 7.41-7.37(\mathrm{~m}, 1 \mathrm{H}), 7.00$ (dd, $J=78.8,2.4 \mathrm{~Hz}, 1 \mathrm{H}), 6.90(\mathrm{~d}, J=2.8 \mathrm{~Hz}, 1 \mathrm{H}), 3.80$ (s, 3H), $3.33(\mathrm{~s}, 3 \mathrm{H}) ;{ }^{13} \mathrm{C}$ NMR $\left(100 \mathrm{MHz}, \mathrm{CDCl}_{3}\right): \delta 166.5$, 162.5, 161.3, 143.2, 141.8, 136.0, 132.8, 130.1, 127.6, 127.3, 127.0, 126.8, 122.6, 120.1, 115.9, 114.4, 55.7, 51.7; HRMSESI (m/z): $[\mathrm{M}+\mathrm{H}]^{+}$Found 294.1114 and calculated 294.1130 for $\mathrm{C}_{18} \mathrm{H}_{16} \mathrm{NO}_{3}$.

\subsection{Synthesis of 1-azabenzanthrone (A)}

2-(Isoquinolin-1-yl)benzoic acid 2a (100 mg, $0.4 \mathrm{mmol}$ ) was poured slowly into concentrated sulfuric acid $(2 \mathrm{~mL})$ at $90^{\circ} \mathrm{C}$. The mixture was stirred and heated at $230-240^{\circ} \mathrm{C}$ for $2 \mathrm{~h}$. After being cooled, the solution was poured onto crushed ice. Sodium hydroxide was added until $\mathrm{pH}=3$ was obtained and the resultant precipitate was filtered off and washed in turn with dilute aqueous sodium hydroxide and water to give the crude product, which was extracted with acetic acid. The extract was condensed under reduced pressure and the resultant precipitate was washed off, dried to give the product 7H-dibenzo[de,h]quinolin-7-one $\mathbf{A}$ as light yellow solid. 51 $\mathrm{mg}, 55 \%$ yield, M.p. $182-184^{\circ} \mathrm{C}$ (lit. $\left.{ }^{8} 181-183^{\circ} \mathrm{C}\right)$, IR ( $\mathrm{KBr}$, $\mathrm{cm}^{-1}$ ): 3055, 2924, 2849, 1657, 1586, 1434, 1361, 1288, 796, 699; ${ }^{1} \mathrm{H}$ NMR (400 MHz, $\left.\mathrm{CDCl}_{3}\right): \delta 8.90(\mathrm{dd}, J=7.6,0.8$ $\mathrm{Hz}, 1 \mathrm{H}), 8.78(\mathrm{~d}, J=5.6 \mathrm{~Hz}, 1 \mathrm{H}), 8.66(\mathrm{dd}, J=7.2,1.2$ $\mathrm{Hz}, 1 \mathrm{H}), 8.42$ (dd, $J=7.8,1.2 \mathrm{~Hz}, 1 \mathrm{H}), 8.15$ (dd, $J=8.4$, $0.8 \mathrm{~Hz}, 1 \mathrm{H}), 7.93-7.89(\mathrm{~m}, 1 \mathrm{H}), 7.81(\mathrm{td}, J=8.0,1.2 \mathrm{~Hz}$, $1 \mathrm{H}), 7.75(\mathrm{~d}, J=5.6 \mathrm{~Hz}, 1 \mathrm{H}), 7.65(\mathrm{td}, J=8.0,1.2 \mathrm{~Hz}$, $1 \mathrm{H}) ;{ }^{13} \mathrm{C}$ NMR $\left(100 \mathrm{MHz}, \mathrm{CDCl}_{3}\right): \delta 183.4,148.8,144.1$, 136.8, 135.2, 134.1, 133.4, 132.4, 130.6, 130.4, 129.9, 129.1, 127.6, 125.3, 122.9, 121.0; HRMS-ESI (m/z): $[\mathrm{M}+\mathrm{H}]^{+}$Found 232.0770 and calculated 232.0762 for $\mathrm{C}_{16} \mathrm{H}_{10} \mathrm{NO}$.

\subsection{Synthesis of 3-bromo-1-azabenzanthrone (B)}

To the solution of 1-azabenzanthrone A (40 mg, $17.3 \mathrm{mmol})$ in $\mathrm{CH}_{3} \mathrm{CN}$ (2.5 mL) was added drop wise with stirring to a solution of $0.5 \mathrm{M} \mathrm{Br}_{2}$ in $\mathrm{CH}_{3} \mathrm{CN}(3.0 \mathrm{~mL}, 1.54 \mathrm{mmol})$. The mixture was heated at $80^{\circ} \mathrm{C}$ for $24 \mathrm{~h}$. The solvent and excess reagent were removed by evaporation under reduced pressure and the residue was chromatographed on silica gel, eluting with $\mathrm{CH}_{2} \mathrm{Cl}_{2}$, to afford 3-bromo-1-aza-benzo[de] anthracen7-one $\mathbf{B}$ as yellow solid. $18 \mathrm{mg}, 34 \%$ yield, M.p. $254-256^{\circ} \mathrm{C}$ (lit. $\left.{ }^{17} 256^{\circ} \mathrm{C}\right), \operatorname{IR}\left(\mathrm{KBr}, \mathrm{cm}^{-1}\right): 3064,2921,2854,1665,1594$, $1559,1472,1388,1278,793 ;{ }^{1} \mathrm{H} \mathrm{NMR}\left(400 \mathrm{MHz}, \mathrm{CDCl}_{3}\right): \delta$ $8.91(\mathrm{~s}, 1 \mathrm{H}), 8.81(\mathrm{~d}, J=8.0 \mathrm{~Hz}, 1 \mathrm{H}), 8.70(\mathrm{dd}, J=7.6,0.8$ $\mathrm{Hz}, 1 \mathrm{H}), 8.47$ (dd, $J=8.4,0.8 \mathrm{~Hz}, 1 \mathrm{H}), 8.39$ (dd, $J=8.0$, $0.8 \mathrm{~Hz}, 1 \mathrm{H}), 8.00(\mathrm{dd}, J=8.4,7.6 \mathrm{~Hz}, 1 \mathrm{H}), 7.82-7.78(\mathrm{~m}$, 1H), 7.68-7.64 (m, 1H); ${ }^{13} \mathrm{C}$ NMR $\left(100 \mathrm{MHz}, \mathrm{CDCl}_{3}\right): \delta$ 183.0, 148.1, 145.8, 136.3, 134.4, 134.2, 132.7, 132.0, 131.7, $130.8,129.4,127.8,125.5,120.2,117.3,116.1$; HRMS-ESI
Table 1. Optimization of the reaction conditions ${ }^{\mathrm{a}}$.

$\begin{array}{llll}\text { Entry } & \mathrm{I}_{2} \text { (equiv.) } & \text { Solvent } & \text { Yient }(2 \mathrm{~mL}), 100{ }^{\circ} \mathrm{C}, \\ \text { sealed tube, } 12 \mathrm{~h}\end{array}$

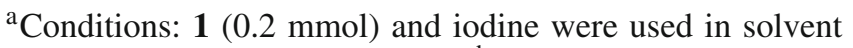
$(2 \mathrm{~mL})$ for $12 \mathrm{~h}$ in sealed tube. ${ }^{\mathrm{b}}$ Isolated yield. ${ }^{\mathrm{c}}$ Nitrogen atmosphere without sealed tube. ${ }^{\mathrm{d}}$ Oxygen atmosphere without sealed tube. ${ }^{e}$ Nitrogen atmosphere in closed reaction tube. ${ }^{\mathrm{f}}$ Oxygen atmosphere in closed reaction tube. ${ }^{\mathrm{g}}$ In sealed tube for $5 \mathrm{~h}$. ${ }^{\mathrm{h}}$ In sealed tube for $8 \mathrm{~h}$.

$(\mathrm{m} / \mathrm{z}):[\mathrm{M}+\mathrm{H}]^{+}$Found 309.9874 and calculated 309.9868 for $\mathrm{C}_{16} \mathrm{H}_{9} \mathrm{BrNO}$.

\subsection{Synthesis of 5-methoxy-6-hydroxy- 1-azabenzanthrone (C)}

The substrate 2-(6,7-dimethoxyisoquinolin-1-yl)benzoic acid 2c $(45 \mathrm{mg}, 0.11 \mathrm{mmol})$ in polyphosphoric acid washeated at $130^{\circ} \mathrm{C}$ for $2 \mathrm{~h}$. The reaction mixture was cooled to room temperature, the mixture was poured into ice water, made alkaline with $10 \%$ aqueous $\mathrm{NH}_{4} \mathrm{OH}$ solution, and extracted with $\mathrm{CH}_{2} \mathrm{Cl}_{2}(5 \times 5 \mathrm{~mL})$, The organic extracts were washed with water, dried over $\mathrm{Na}_{2} \mathrm{SO}_{4}$ and solvent was removed by evaporation under reduced pressure and the residue was chromatographed on silica gel, eluting with $\mathrm{CH}_{2} \mathrm{Cl}_{2}$, to afford 6-hydroxy-5-methoxy-1-aza-benzo[de]anthracen-7-one $\mathbf{C}$ as yellow solid. $23 \mathrm{mg}, 57 \%$ yield, M.p. $233-235^{\circ} \mathrm{C}$ (lit. ${ }^{17} 233-$ $\left.234^{\circ} \mathrm{C}\right), \operatorname{IR}\left(\mathrm{KBr}, \mathrm{cm}^{-1}\right): 3398,2926,2843,1726,1624,1574$, 1489, 1276, 1090, 927, 753; ${ }^{1} \mathrm{H}$ NMR $\left(400 \mathrm{MHz}, \mathrm{CDCl}_{3}\right): \delta$ 16.05 (s, 1H), 9.09 (d, $J=8.0 \mathrm{~Hz}, 1 \mathrm{H}), 8.81(\mathrm{~d}, J=5.2 \mathrm{~Hz}$, $1 \mathrm{H}), 8.57$ (dd, $J=8.0,1.2 \mathrm{~Hz}, 1 \mathrm{H}), 7.92(\mathrm{td}, J=8.0,1.2$ $\mathrm{Hz}, 1 \mathrm{H}), 7.76-7.72(\mathrm{~m}, 1 \mathrm{H}), 7.68(\mathrm{~d}, J=5.6 \mathrm{~Hz}, 1 \mathrm{H}) 7.35$ $(\mathrm{s}, 1 \mathrm{H}), 4.12(\mathrm{~s}, 3 \mathrm{H}) ;{ }^{13} \mathrm{C} \mathrm{NMR}\left(100 \mathrm{MHz}, \mathrm{CDCl}_{3}\right): \delta 185.1$, 164.7, 153.1, 144.2, 143.7, 139.4, 134.2, 131.1, 130.4, 129.7, 


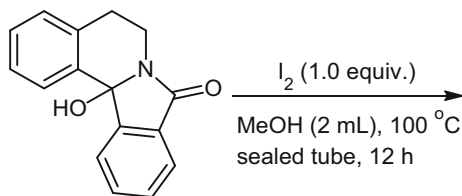

1

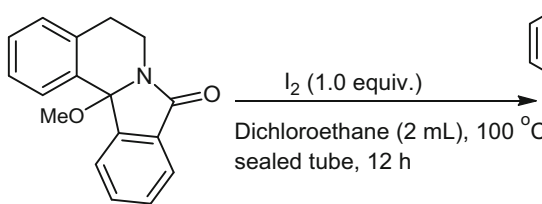

$3,92 \%$

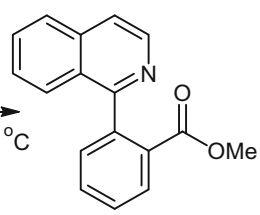

4, $53 \%$

Scheme 2. Synthesis of methyl 2-(isoquinolin-1-yl)benzoate 4.

Table 2. Iodine mediated etherification of substituted isoindoloisoquinolinones ${ }^{\mathrm{a}}$.

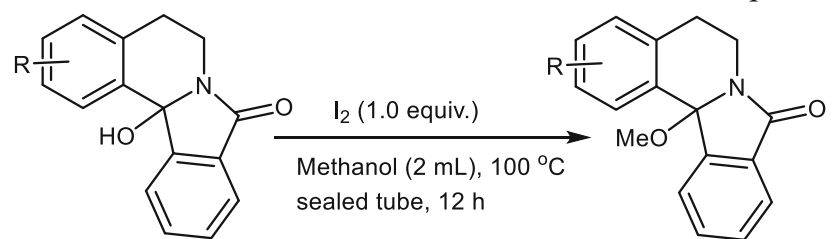

1a-1c, 1e-1h, 1j

$3 a-3 c, 3 e-3 h, 3 j$<smiles>[B]C12C(=O)C(OC)(c3ccccc31)c1ccccc12</smiles>

$3 a, 92 \%$<smiles>COC12c3ccc(Br)cc3CCN1C(=O)c1ccccc12</smiles>

3b, $93 \%$<smiles>COc1cc2c(cc1OC)C1(OC)c3ccccc3C(=O)N1CC2</smiles>

$3 c, 76 \%$<smiles>COc1ccc(OC)c2c1CCN2C(=O)c1ccccc1O</smiles><smiles>COc1cc2c(c(OC)c1OC)CCN1C(=O)c3ccccc3C21OC</smiles><smiles>COC12c3ccc(C)cc3CCN1C(=O)c1ccccc12</smiles>

$3 g, 67 \%$

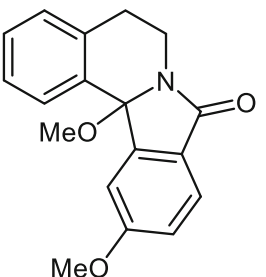

$3 \mathrm{~h}, 39 \%$<smiles>COC12c3cc4c(cc3CCN1C(=O)c1ccccc12)OCO4</smiles>

3j, $67 \%$

${ }^{\mathrm{a} C}$ Conditions: In all reactions, $0.4 \mathrm{mmol}$ of $\mathbf{1}$ was used.

$126.6,125.4,120.7,114.2,112.1,108.8,56.5$; HRMS-ESI $(\mathrm{m} / \mathrm{z}):[\mathrm{M}+\mathrm{H}]^{+}$Found 278.0820 and calculated 278.0817 for $\mathrm{C}_{17} \mathrm{H}_{12} \mathrm{NO}_{3}$.

\section{Results and Discussion}

For preliminary studies, the compound $\mathbf{1}$ was chosen as a model substrate. To exploit the oxidizing ability of iodine, the isoindoloisoquinolinone derivative $\mathbf{1}$ was treated with 1.0 equivalent of $\mathrm{I}_{2}$ at room temperature in 1,2-dichloroethane. Even after $24 \mathrm{~h}$, the substrate $\mathbf{1}$ failed to generate the expected product 2 . Performing the reaction at $60^{\circ} \mathrm{C}$ for $12 \mathrm{~h}$ as well as $24 \mathrm{~h}$ with 1.0 equivalent of $I_{2}$ failed to furnish the product 2. Quite interestingly, the product 2 was obtained in $28 \%$ and $32 \%$ yield, respectively, when the experiments were carried out at $100^{\circ} \mathrm{C}$ under $\mathrm{N}_{2}$ as well as $\mathrm{O}_{2}$ atmosphere. On the other hand, when the reactions were performed in a closed vessel at $100^{\circ} \mathrm{C}$ under $\mathrm{N}_{2}$ as well as $\mathrm{O}_{2}$ atmosphere for $12 \mathrm{~h}$, the yields of the product 2 increased to $41 \%$ and $38 \%$, respectively (Table 1 , entries 3,4 ). These 
Table 3. Iodine mediated oxidative dehydrogenation of substituted isoindoloisoquinolinones ${ }^{\mathrm{a}}$.

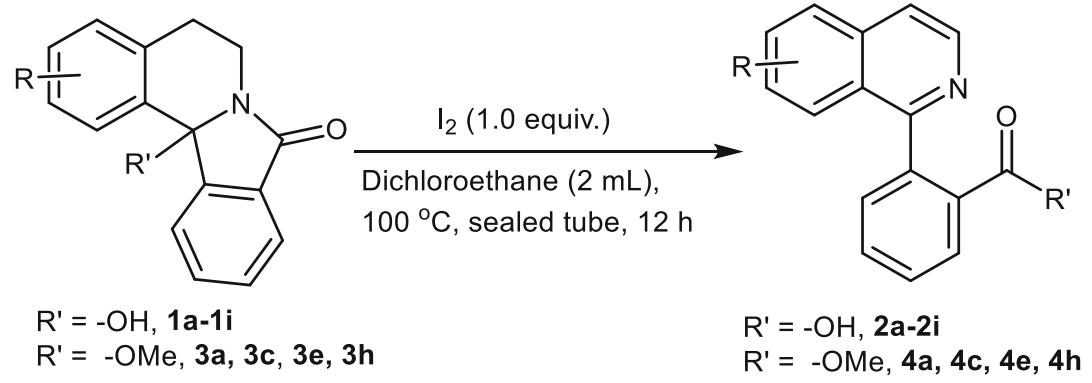<smiles>C/C=C(/C)C(=O)O</smiles>
$2 e, 45 \%$<smiles>COc1cc2c(-c3ccccc3C(=O)O)nccc2c(OC)c1OC</smiles>
$2 c, 75 \%$<smiles>COc1cc(OC)c2c(-c3ccccc3)nccc2c1</smiles><smiles>O=C(O)c1ccccc1-c1nccc2cc3ccccc3cc12</smiles>

$2 \mathrm{i}, 41 \%$<smiles>CC(=O)c1ccccc1-c1nccc2ccccc12</smiles>

$4 a, 53 \%$<smiles>Cc1ccc2c(-c3ccccc3C(=O)O)nccc2c1</smiles>

2g, $53 \%$<smiles>COc1ccc(C(=O)O)c(-c2nccc3ccccc23)c1</smiles>

2h, $76 \%$<smiles>COc1ccc(OC)c2c(-c3ccccc3C(C)=O)nccc12</smiles>

$4 e, 46 \%$<smiles>COc1cc2ccnc(-c3ccccc3C(C)=O)c2cc1OC</smiles>

4c, $42 \%$<smiles>COc1ccc(C(C)=O)c(-c2nccc3ccccc23)c1</smiles>

4h, $61 \%$

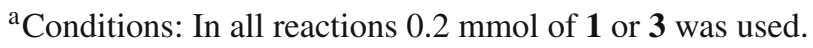

experiments prompted us to carry out this reaction in a sealed tube. Therefore, the compound $\mathbf{1}$ was first treated with $20 \mathrm{~mol} \%$ of $\mathrm{I}_{2}$ in 1,2-dichloroethane at $100^{\circ} \mathrm{C}$ in a sealed tube which failed to generate the expected product 2 (Table 1, entry 5). Increasing the amount of iodine to $40 \mathrm{~mol} \%$ was also not fruitful (Table 1, entry 6). The compound 2 was obtained in $10 \%$ and $15 \%$ yields when the reaction was carried out with 60 mol\% and 80 mol\% of $\mathrm{I}_{2}$, respectively (Table 1, entries 7, 8). Later, the reaction was carried out with 1.0 equivalent of iodine, which afforded the desired product $\mathbf{2}$ in $92 \%$ yield (Table 1, entry 9). Increasing the amount of iodine from 1.0 to 2.0 equivalents too decreased the yield from $92 \%$ to $70 \%$ (Table 1, entry 10). Decreasing the time from 12 


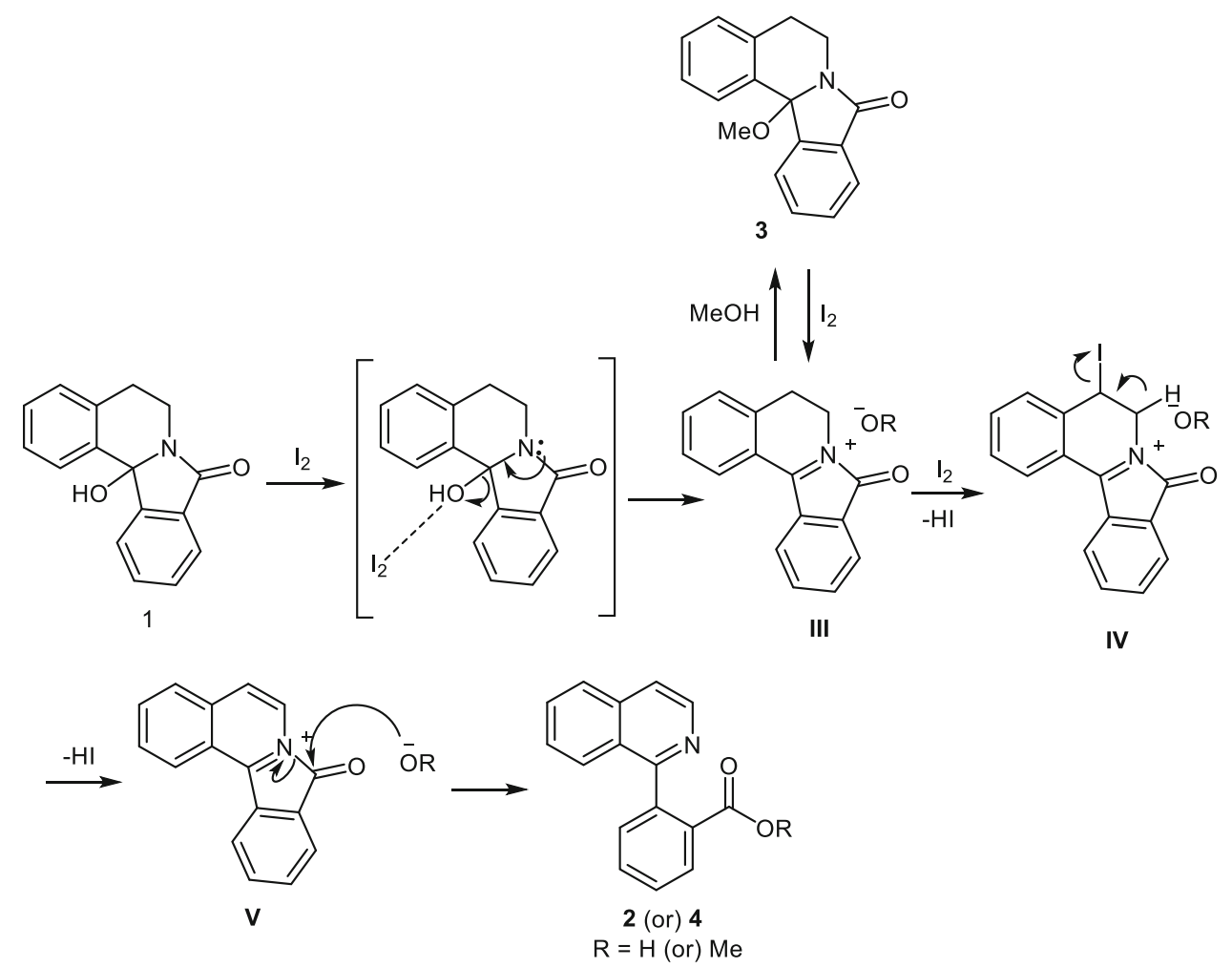

Scheme 3. Plausible mechanism for the formation of $\mathbf{3}$ and $\mathbf{2}$ (or) 4.

$\mathrm{h}$ to $5 \mathrm{~h}$ as well as to $8 \mathrm{~h}$ with 2.0 equivalents of iodine gave the product 2 in $53 \%$ and $80 \%$ yield, respectively (Table 1, entries 11, 12). Based on these observations it is concluded that, 1.0 equivalent of iodine is suitable for obtaining the desired product $\mathbf{2}$ in good yield.

With 1.0 equivalent of $\mathrm{I}_{2}$, experiments were carried out in different solvents such as toluene, tetrachloroethane, acetonitrile, DMF and DMSO at $100^{\circ} \mathrm{C}$ in sealed tube. In the case of nonpolar solvents such as toluene and tetrachloroethane, the compound $\mathbf{2}$ was obtained in $87 \%$ and $89 \%$ yield, respectively (Table 1 , entries 13, 14). The solvents such as $\mathrm{CH}_{3} \mathrm{CN}, \mathrm{DMF}$ and DMSO failed to produce 2 (Table 1, entries 15-17). From these systematic observations, the optimized condition for the conversion of $\mathbf{1}$ to $\mathbf{2}$ was found to be 1.0 equivalent of iodine in 1,2 -dichloroethane at $100^{\circ} \mathrm{C}$ in a sealed tube for $12 \mathrm{~h}$ (Table 1 ).

With optimized reaction condition in methanol in lieu of 1,2-dichloroethane, the compound $\mathbf{1}$ failed to furnish the product 2; instead, ether $\mathbf{3}$ was produced in $92 \%$ yield. Subsequently, when this compound $\mathbf{3}$ was subjected to the oxidative dehydrogenation condition using $\mathrm{I}_{2}$, the ester 4 was generated in 53\% yield (Scheme 2).

This observation supports the involvement of internal nucleophile either $\mathrm{HO}^{-}$or $\mathrm{MeO}^{-}$present in the substrate $\mathbf{1}$ or $\mathbf{3}$ is responsible for the formation of acid $\mathbf{2}$ or ester 4. To prove this, when the substrate 1 was treated with iodine in the presence of external $\pi$-nucleophile, i.e., anisole, the reaction furnished only acid $\mathbf{2}$ in $86 \%$ yield.

To further generalize this etherification reaction, different types of methoxy/methyl/bromo substituted isoindoloisoquinolinones $(\mathbf{1 a - 1 c}, \mathbf{1 e - 1 h}, \mathbf{1 j})$ were treated with iodine under the optimized etherification conditions. As illustrated in Table 2, for example, unsubstituted 1a, 3,4-dimethoxy 1c, 2,5-dimethoxy 1e and 2,3,4-trimethoxy $\mathbf{1 f}$ isoindoloisoquinolinone derivatives successfully furnished the corresponding ethers $\mathbf{3 a}, \mathbf{3 c}$, $\mathbf{3 e}$ and $\mathbf{3 f}$ in moderate to good yields. Similarly, the 3-bromo derivative $\mathbf{1 b}$ also produced the ether $\mathbf{3 b}$ in 93\% yield. In addition, 3-methyl derivative $\mathbf{1 g}$ and methylenedioxy derivative $\mathbf{1} \mathbf{j}$ also delivered the corresponding ether, $\mathbf{3 g}$ and $\mathbf{3 j}$ in moderate yield. The derivative $\mathbf{1 h}$ also delivered the corresponding ether $\mathbf{3 h}$ in $39 \%$ yield.

With the optimized reaction conditions in hand, we then evaluated the scope of the oxidative dehydrogenation and ring opening of lactam using a series of isoindoloisoquinolinone derivatives (1a-1i) and the results are summarized in Table 3. All the substrates underwent oxidative dehydrogenation and ring opening reactions smoothly and delivered the corresponding products $\mathbf{2 a - 2 i}$. For example, unsubstituted 1a, 3-bromo 1b, 3,4-dimethoxy 1c and 3,5-dimethoxy 1d derivatives delivered products $\mathbf{2 a - 2 c}$ in moderate to good yields. 
<smiles>CC(C)[C@H]1CC[C@@H](C)C1=O</smiles>

1a, $78 \%$<smiles>O=C1c2ccccc2-c2ncc(Br)c3cccc1c23</smiles>

B, $34 \%$

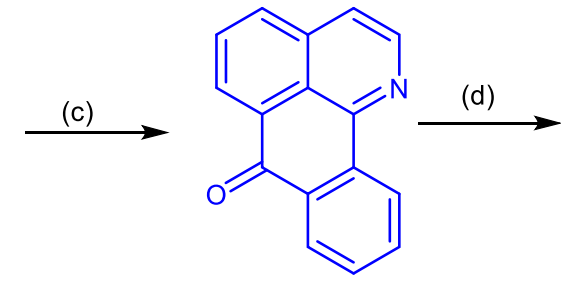

A, $55 \%$<smiles>O=C(O)c1ccccc1-c1nccc2ccccc12</smiles>

2a, $92 \%$

a) $\mathrm{TfOH}, \mathrm{CH}_{2} \mathrm{Cl}_{2}, 0^{\circ} \mathrm{C}$-rt, $1 \mathrm{~h}$, aq. $\mathrm{NaHCO}_{3}, 1 \mathrm{~h}$; b) $\mathrm{I}_{2}$, dichloroethane, sealed tube, $100^{\circ} \mathrm{C}, 12 \mathrm{~h}$; c) $\mathrm{H}_{2} \mathrm{SO}_{4}, 90^{\circ} \mathrm{C}, 1 \mathrm{~h}$ then $220^{\circ} \mathrm{C}, 2 \mathrm{~h}$; d) $\mathrm{Br}_{2}, \mathrm{CH}_{3} \mathrm{CN}, 80^{\circ} \mathrm{C}, 24 \mathrm{~h}$.<smiles>COc1ccc(CCN2C(=O)c3ccccc3C2=O)cc1OC</smiles><smiles>COc1cc2c(cc1OC)C1(O)c3ccccc3C(=O)N1CC2</smiles>

1c, $96 \%$<smiles>COc1cc2ccnc(-c3ccccc3C(=O)O)c2cc1OC</smiles>

2c, $75 \%$<smiles></smiles>

C, $57 \%$

a) $\mathrm{TfOH}, \mathrm{CH}_{2} \mathrm{Cl}_{2}, 0^{\circ} \mathrm{C}$-rt, $30 \mathrm{~min}$, aq. $\mathrm{NaHCO}_{3}, 1 \mathrm{~h}$; b) $\mathrm{I}_{2}$, dichloroethane, sealed tube, $\left.100^{\circ} \mathrm{C}, 12 \mathrm{~h} ; \mathrm{c}\right) \mathrm{PPA}, 130^{\circ} \mathrm{C}, 3 \mathrm{~h}$.

Scheme 4. Synthesis of 1-azabenzanthrone A, 3-bromo-1-azabenzanthrone B and 5-methoxy-6-hydroxy-1-azabenzanthrone $\mathbf{C}$.

Further, 2,5-dimethoxy $\mathbf{1 e}, \mathbf{1 h}$ and naphthyl $\mathbf{1 i}$ derivatives under standard conditions afforded the products $\mathbf{2 e}$, $\mathbf{2 h}$ and $\mathbf{2 i}$ in $45 \%, 76 \%$ and $41 \%$ yields, respectively. Formation of these products was characterized by IR, NMR and HRMS analysis, except 2,3,4-trimethoxy 1f, 3methyl 19 derivatives. Though the reactions were clean, the spectral analysis after column chromatography or recrystallization, especially ${ }^{13} \mathrm{C} \mathrm{NMR}$, of the products obtained from $\mathbf{1 f}$ and $\mathbf{1 g}$, were not clear. This may be due to the inherent poor stability of these molecules. But the products were confirmed by IR, ${ }^{1} \mathrm{H}$ NMR and mass spectrometry.

To overcome this problem, the compounds 1 ff and $1 \mathrm{~g}$ were subjected to etherification followed by oxidative cleavage conditions. Etherification of the compounds 1 f and $1 \mathrm{~g}$ underwent smoothly and afforded the products $\mathbf{3 f}$ and $\mathbf{3 g}$ in $66 \%$ and $67 \%$ yields, respectively. Oxidative dehydrogenation and ring opening reaction 
of these ethers $\mathbf{3 f}$ and $\mathbf{3 g}$ furnished the ester products 4f and $\mathbf{4 g}$. Unfortunately, again the isolation of these products has become difficult due to the instability of products. Oxidative dehydrogenation and ring opening strategy has been extended to ethers such as $\mathbf{3 a}, \mathbf{3 c}, \mathbf{3 e}$ and $\mathbf{3 h}$ (Table 3 ). Under the optimized conditions, these substrates furnished the expected products $\mathbf{4 a}, \mathbf{4 c}, \mathbf{4 e}$ and $4 \mathbf{h}$ in $42-61 \%$ yields.

The formation of ether $\mathbf{3}$ from alcohol $\mathbf{1}$ may be explained through the Lewis acidic nature of iodine. Coordination of iodine with oxygen lone pair in $\mathrm{OH}$ group may facilitate the formation of condensed cyclic $N$-acyliminium ion which on further reaction with methanol might have generated the corresponding ether $\mathbf{3}$ (Scheme 3). The formation of $\mathbf{2}$ or $\mathbf{4}$ may follow the similar mechanism until the formation of condensed cyclic $N$-acyliminium ion III from either $\mathbf{1}$ or $\mathbf{3}$ in presence of $I_{2}$. In the absence of nucleophilic solvent methanol, the condensed cyclic $N$-acyliminium ion III may have possibly undergone benzylic iodination to $\mathbf{I V}$, followed by dehydroiodination through 1,4-elimination, which may lead to the formation of highly electrophilic isoquinolium imide $\mathbf{V}$. The electrophilic carbonyl group in $\mathbf{V}$ might have accepted the available nucleophiles $\mathrm{HO}^{-}$or $\mathrm{MeO}^{-}$generated in the reaction to deliver the ring opened products either 2 or 4 (Scheme 3).

To show the utility of this protocol, we intended to synthesize azabenzanthrones and analogue of menisporphine. Accordingly, 1-azabenzanthrone A from phenethylphthalimide was synthesized in 3 steps with $40 \%$ overall yield (Scheme 4). Bromination of 1azabenzanthrone successfully furnished 3-bromo-1-azabenzanthrone B in 34\% yield. 5-Methoxy-6-hydroxy1-azabenzanthrone $\mathbf{C}$, which is an analogue of 6-Odemethyl menisporphine, was successfully synthesized from 3,4-dimethoxy phenethylphthalimide in 3 steps with $41 \%$ overall yield (Scheme 4). Compound $\mathbf{C}$ is a potential precursor for the synthesis of Lakshminine, an alkaloid extracted from a basic fraction of woody vines (collected from two bush-ropes) of Sciadotenia toxifera. ${ }^{15}$

\section{Conclusions}

In conclusion, we have developed a simple methodology for the synthesis of functionalized 1-aryl isoquinolines which are useful intermediates for the synthesis of 1-azabenzanthrone derivatives. In addition, we have synthesized 1-azabenzanthrone A, 3-bromo1-azabenzanthrone $\mathbf{B}$ and 5-methoxy-6-hydroxy-1-azab enzanthrone C. 5-Methoxy-6-hydroxy-1-azabenzanthrone $\mathbf{C}$, can be utilized for the synthesis of
Lakshminine. This method could be utilized for the synthesis of analogues of menisporphine alkaloids and 1-aryl-1,2,3,4-tetrahydroisoquinolines.

\section{Supplementary Information (SI)}

${ }^{1} \mathrm{H}$ and ${ }^{13} \mathrm{C}$ NMR spectra of the ethers $\mathbf{3 a}-\mathbf{3 c}, \mathbf{3 e -} \mathbf{3 h}, \mathbf{3 j}$, acids/esters $\mathbf{2 a - 2 i / 4 a , ~ 4 c , ~ 4 e , ~} \mathbf{4 h}$ and compounds $\mathrm{A}, \mathbf{B}, \mathbf{C}$ are provided in Supplementary Information which is available at www.ias.ac.in/chemsci.

\section{Acknowledgements}

We thank DST and CSIR, New Delhi, India for financial support. K.M.M.A. thanks UGC, New Delhi for SRF. We gratefully acknowledge the CIF, Pondicherry University for NMR/IR data. DST-FIST program, Department of Chemistry, Pondicherry University is gratefully acknowledged for HRMS. We also acknowledge UGC for the award of SAP (DSA) program to the Department of Chemistry, Pondicherry University. We acknowledge IPLS program, Pondicherry University. Funding was provided by Science and Engineering Research Board (Grant No. SR/S1/OC-04/2011(G); Dated: 25/08/2011), Council of Scientific and Industrial Research (Grant No. 02(0027)/11/EMR-II).

\section{References}

1. Yu B W, Meng L H, Chen J Y, Zhou T X, Cheng K F, Ding J and Qin G W 2001 Cytotoxic Oxoisoaporphine Alkaloids from Menispermumdauricum J. Nat. Prod. 64 968

2. (a) Tang $H$, Ning $F X$, Wei $Y$ B, Huang $S$ L, Huang Z S, Chan A S C and Gu L Q 2007 Derivatives of oxoisoaporphine alkaloids: A novel class ofselective acetylcholinesterase inhibitors Bioorganic Med. Chem. Lett. 17 3765; (b) Tang H, Wei Y B, Zhang C, Ning F X, Qiao W, Huang S L, Ma L, Huang Z S and Gu L Q 2009 Synthesis, biological evaluation and molecular modeling of oxoisoaporphine and oxoaporphine derivatives as new dual inhibitors of acetylcholinesterase/butyrylcholinesterase Eur. J. Med. Chem. 442523

3. Tang H, Wang X D, Wei Y B, Huang S L, Huang Z S, Tan J H, An L K, Wu J Y, Chan A S C and Gu L Q 2008 Oxoisoaporphine alkaloid derivatives: Synthesis, DNA binding affinity and cytotoxicity Eur. J. Med. Chem. 43 973

4. (a) Dong X, Mondranondra I O, Che C T, Fong H H S and Farnsworth N R 1989 Kmeriol and Other Aromatic Constituents of Kmeria Duperreana Pharm. Res. 6 637; (b) Warthen D, Gooden E L and Jacobson M 1969 Tumor Inhibitors: Liriodenine, a Cytotoxic Alkaloid from Annona glabra J. Pharm. Sci. 58637

5. Castro-Castillo V, Rebolledo-Fuentes M, Theoduloz C and Cassels B K 2010 Synthesis of Lakshminine and Antiproliferative Testing of Related Oxoisoaporphines J. Nat. Prod. 731951 
6. Castro-Castillo V, Suárez-Rozas C, Pabón A, Pérez E G, Cassels B K and Blair S 2013 Synthesis and antiplasmodial activity of some 1-azabenzanthrone derivatives Bioorg. Med. Chem. Lett. 23327

7. Chen Z F, Qin Q P, Qin J L, Liu Y C, Huang K Bin, Li Y L, Meng T, Zhang G H, Peng Y, Luo X J and Liang H 2015 Stabilization of G-Quadruplex DNA, Inhibition of Telomerase Activity, and Tumor Cell Apoptosis by Organoplatinum(II) Complexes with Oxoisoaporphine J. Med. Chem.58 2159

8. Iwashima S, Ueda T, Honda H, Tsujioka T and Ohno M 1984 Synthesis and Physical Properties of Azapolycyclic Hydrocarbons. Part 1. Preparation of 1-Azabenzanthrone and its Condensation Products and their Structural Determination J. Chem. Soc. Perkin. Trans. I 72177

9. (a) Kunitomo J and Satoh M 1982 Strucure of Menisporphine: A new type of isoquinoline alkaloid Chem. Pharm. Bull. 30 2659; (b) Kunitomo J and Satoh M 1983 Structure and synthesis of menisporphine, a new type of isoquinoline alkaloid Tetrahedron 39 3261; (c) Kunitomo J, Kaede S and Satoh M 1985 The structure of 2,3-Dihydromenisporphine and the Synthesis of Dauriporphine, Oxoisoaporphine Alkaloids from Menispermum dauricum DC Chem. Pharm. Bull. 33 2778

10. Sobarzo-Sánchez E, Cassels B K, Jullian C and Castedo L 2003 Complete structural and spectral assignment of oxoisoaporphines by HMQC and HMBC experiments Magn. Reson. Chem. 41296

11. Tekale U S, Kauthale S S, Dake S A,Sarda S R and Pawar R P 2012 Molecular Iodine: An Efficient and Versatile Reagent for Organic Synthesis Curr. Org. Chem. 161485

12. Mphahlele M J 2009 Molecular Iodine-An Expedient Reagent for Oxidative Aromatization Reactions of $\alpha, \beta$ Unsaturated Cyclic Compounds Molecules 145308

13. Arun Kumar R, Uma Maheswari C, Satheesh G, Jyothi C and Rajender Reddy K 2011 Synthesis of 3HQuinazolin-4-ones and 4H-3,1-Benzoxazin-4-ones via Benzylic Oxidation and Oxidative Dehydrogenation using Potassium Iodide-tert-Butyl Hydroperoxide $A d v$. Synth. Catal. 353401
14. (a) Selvakumar J, Makriyannis A and Ramanathan C R 2010 An unusual reactivity of $\mathrm{BBr}_{3}$ : Accessing tetrahydroisoquinoline units from N-phenethylimides Org. Biomol. Chem. 8 4056; (b) Selvakumar J and Ramanathan C R 2011 Brønsted acid assisted activation of imide carbonyl group: regioselective synthesis of isoindoloisoquinolinone alkaloid $( \pm)$-nuevamine Org. Biomol. Chem. 9 7643; (c) Mangalaraj S and Ramanathan C R 2012 Construction of tetrahydro$\beta$-carboline skeletons via Brønsted acid activation of imide carbonyl group: syntheses of indole alkaloids $( \pm)$ harmicine and $( \pm)$-10-desbromoarborescidine-A RSC $A d v .2$ 12665; (d) Selvakumar J, Rao R S, Srinivasapriyan V, Marutheeswaran S and Ramanathan C R 2015 Synthesis of Condensed Tetrahydroisoquinoline Class of Alkaloids by Employing TfOH-Mediated Imide Carbonyl Activation Eur. J. Org. Chem. 2175; (e) Mangalaraj S, Selvakumar J and Ramanathan C R 2015 Syntheses of fused tetrahydro- $\beta$-carboline analogues through imide carbonyl activation using $\mathrm{BBr}_{3}$ : Evidence for the involvement of fused cyclic $\mathrm{N}$-acyliminium ion intermediate J. Chem. Sci. 127 811; (f) Selvakumar J, Mangalaraj S, Achari K M M, Mukund K and Ramanathan C R 2017 Triflic Acid Mediated Cyclization of Unsymmetrical N-Phenethyl and N-(3-Indolylethyl)succinimides: Regio- and Diastereoselective Synthesis of Substituted Pyrroloisoquinolinones and Indolizinoindolones Synthesis 491053

15. Killmer L, Vogt F G, Freyer A J, Menachery M D and Adelman C M 2003 Lakshminine, a New Rare Oxoisoaporphine Alkaloid from Sciadotenia toxifera, and Structural Revisions of Telazoline and Teladiazoline, Two Related Oxoaporphines from Telitoxicum peruvianum and T. glaziovii J. Nat. Prod. 66115

16. Armarego W L F and Chai C L L 2006 Purification of Laboratory Chemicals $6^{\text {th }}$ ed. (Oxford: Elsevier)

17. Ueda T, Abliz Z, Sato M, Nishimura M, Iwashima S, Aoki J, Kan T, Matsunaga S and Tanaka R 1990 Structure determination by MS, NMR and UV spectra of bromo and nitro derivatives of 1-azabenzanthrone J. Mol. Struct. 224313 